\title{
EXISTENCE OF NON-SUBNORMAL POLYNOMIALLY HYPONORMAL OPERATORS
}

\author{
RAÚL E. CURTO AND MIHAI PUTINAR
}

\section{INTRODUCTION}

In 1950, P. R. Halmos, motivated in part by the successful development of the theory of normal operators, introduced the notions of subnormality and hyponormality for (bounded) Hilbert space operators. An operator $T$ is subnormal if it is the restriction of a normal operator to an invariant subspace; $T$ is hyponormal if $T^{*} T \geq T T^{*}$. It is a simple matrix calculation to verify that subnormality implies hyponormality, but the converse is false. One reason is that subnormality is invariant under polynomial calculus (indeed, analytic functional calculus), while hyponormality is not. If one then defines $T$ to be polynomially hyponormal when $p(T)$ is hyponormal for every polynomial $p \in \mathbf{C}[z]$, the following question arises naturally.

Main Question. Let $T$ be polynomially hyponormal. Must $T$ be subnormal?

Both the class of subnormal operators and the class of hyponormal operators have been the subject of much investigation during the last thirty years or so, and many important developments in operator theory have dealt with them (e.g., the Berger-Shaw Theorem, the singular integral model, and the 2-subscalar model, for hyponormal operators; S. Brown's proof of the existence of nontrivial invariant subspaces, J. Conway and R. Olin's construction of the functional calculus and J. Thomson's description of the spectral picture in the cyclic case, for subnormal operators, etc.;

\footnotetext{
Received by the editors November 23, 1990 and, in revised form, February 10, 1991.

1980 Mathematics Subject Classification (1985 Revision). Primary 47B20, 47A65, 44A60; Secondary 46A22, 52A07.

Key words and phrases. Subnormal, polynomially hyponormal, cones of polynomials.

The first author was partially supported by a grant from the National Science Foundation and by a University of Iowa Faculty Scholar award.
} 
cf. [Con, Xia, Cla, MP, Put, Tho]). The Main Question appears in the early stages of these theories, at least in weaker or implicit forms [Con, Hal, St]. Therefore, the settling of it becomes an important matter in terms of furthering our knowledge of both classes.

In this note we announce that the answer to the Main Question is negative. As a matter of fact, we show that the answer to a more specific question is also negative. To formulate the latter, we need to recall the notion of joint hyponormality [CMX]. A commuting $k$-tuple $T=\left(T_{1}, \ldots, T_{k}\right)$ of operators on a Hilbert space $\mathscr{H}$ is said to be jointly hyponormal if the joint commutator

$$
\left[T^{*}, T\right]:=\left[\begin{array}{ccc}
{\left[T_{1}^{*}, T_{1}\right]} & \cdots & {\left[T_{k}^{*}, T_{1}\right]} \\
\vdots & & \vdots \\
{\left[T_{1}^{*}, T_{k}\right]} & \cdots & {\left[T_{k}^{*}, T_{k}\right]}
\end{array}\right]
$$

is a positive operator on $\mathscr{H} \oplus \cdots \oplus \mathscr{H}$. An operator $T$ is said to be $k$-hyponormal if $\left(T, \ldots, T^{k}\right)$ is jointly hyponormal. The well-known Bram-Halmos criterion for subnormality (see [Con, III.1.9]) establishes that an operator $T$ is subnormal if and only if $T$ is $k$-hyponormal for every $k \geq 1$ (cf. [CMX, $\mathrm{Cu} 1, \mathrm{Cu} 2]$ ).

Auxiliary Question. Let $T$ be polynomially hyponormal. Must $T$ be 2-hyponormal?

As we shall see below, polynomially hyponormality does not suffice to prove even 2-hyponormality, much less subnormality, thus showing that the class of polynomially hyponormal operators is indeed much larger than that of subnormal operators. In [McCP], the authors establish that to answer the Main Question, it suffices to restrict attention to the class of unilateral weighted shifts, a collection well understood in many respects [Hal, Shi, St]. This led to a rather detailed investigation of $k$-hyponormality and quadratic hyponormality for unilateral weighted shifts $[\mathrm{Cu} 1, \mathrm{CF}]$, with an emphasis on characterizations and model theory. The proof of our main result, however, relies more on a detailed investigation of certain polynomial cones (in the spirit of the classical theory of moments) and, incidentally, it requires that certain linear functionals do not arise from weighted shifts.

CONTRACTIONS AS LINEAR FUNCTIONALS ON POLYNOMIALS

J. Agler developed in [Ag1] and [Ag2] an ordered functional calculus for contractions, which transfers notions associated with operator positivity to positivity for linear functionals on spaces 
of polynomials. To describe this, we need some notation. Given an operator $T$ on $\mathscr{H}$ and a polynomial $p \in \mathbf{C}[z, \bar{z}], p(z, \bar{z})=$ $\sum_{m, n} a_{m n} z^{m} \bar{z}^{n}$, we let $p\left(T, T^{*}\right):=\sum_{m, n} a_{m n} T^{* n} T^{m}$. For $T$ and $\gamma \in \mathscr{H}$ fixed, we define $\Lambda_{T}: \mathbf{C}[z, \bar{z}] \rightarrow \mathbf{C}$ by $\Lambda_{T}(p):=$ $\left(p\left(T, T^{*}\right) \gamma, \gamma\right), p \in \mathbf{C}[z, \bar{z}]$. By combining [McCP, Theorem 2.4] with [Ag2, Theorem 3.3], we can show that the map $(T, \gamma) \rightarrow$ $\Lambda_{T}$ establishes a one-to-one correspondence between the unitary equivalence classes of $k$-hyponormal contractions with fixed cyclic vector $\gamma$ and the linear functionals on $\mathbf{C}[z, \bar{z}]$ which are positive on the cone $\mathscr{S}^{k}$ generated by all the polynomials of the form $\left(1-|z|^{2}\right)|p(z)|^{2}$ and $\left|\sum_{i=0}^{k} q_{i}(z) \bar{z}^{i}\right|^{2}$, where $p, q_{0}, \ldots, q_{k} \in \mathbf{C}[z]$. The same map also establishes a one-to-one correspondence between the polynomially hyponormal contractions with fixed cyclic vector $\gamma$ and the linear functionals positive on the cone $\mathscr{W}$ generated by all the polynomials of the form $\left(1-|z|^{2}\right)|p(z)|^{2}$ and $|\overline{q(z)}+\overline{p(z)} r(z)|^{2}$, where $p, q, r \in \mathbf{C}[z]$.

It follows that in order to answer the Auxiliary Question in the negative, it suffices to exhibit a polynomial $p(z, \bar{z})$ of degree 2 in $\bar{z}$ and a linear functional $\Lambda$ on $\mathbf{C}[z, \bar{z}]$ such that $\Lambda(p)<0$ and $\left.\Lambda\right|_{\mathscr{W}} \geq 0$. This is precisely what we set out to do in the next section.

\section{SEPARATION OF A POLYNOMIAL IN $\mathscr{S}^{2}$ FROM $\mathscr{W}$}

In this section we sketch the proof of our main result.

Theorem. There exists a polynomially hyponormal operator which is not 2-hyponormal.

Sketch of the proof. Let $\Gamma_{m}$ denote the cone generated by polynomials of the form $|\overline{q(z)}+\overline{p(z)} r(z)|^{2}$, where the total degree in $z$ and $\bar{z}$ is at most $m$, and let $\mathscr{W}_{m}$ be the cone generated by $\Gamma_{m}$ and the polynomials of the form $\left(1-|z|^{2}\right) \sum_{j}\left|r_{j}(z)\right|^{2}$, again keeping the total degree at most $m$. Clearly $\mathscr{W}=\bigcup_{m} \mathscr{W}_{m}$. Also, let $\mathbf{C}[z, \bar{z}]_{m}^{h}$ denote the homogeneous polynomials of degree $m$, and for a cone $K$ let $K_{m}^{h}:=K \cap \mathbf{C}[z, \bar{z}]_{m}^{h}$; also, let $\mathbf{R}[x, y]_{m}$ (resp. $\mathbf{R}[x, y]_{m}^{h}$ ) denote the real polynomials (resp. homogeneous polynomials) of degree at most $m$ (resp. $m$ ).

Step 1. $\left\{p(z, \bar{z}) \in \mathbf{C}[z, \bar{z}]_{m}^{h}: p=\bar{p}\right\}=\mathbf{R}[x, y]_{m}^{h}$, via the usual identification $\frac{z+\bar{z}}{2}=x, \frac{z-\bar{z}}{2 i}=y$. 
Step 2. $\mathbf{R}[x, y]_{m}=\mathscr{W}_{m}-\mathscr{W}_{m}$ for every $m \geq 1$ and $\mathbf{R}[x, y]_{m}^{h}=$ $\Gamma_{m}^{h}-\Gamma_{m}^{h}=\mathscr{W}_{m}^{h}-\mathscr{W}_{m}^{h}$ for every even $m \geq 2$, so in particular int. $\mathscr{W}_{m} \neq \varnothing(m \geq 1)$ and int. $\Gamma_{m}^{h} \neq \varnothing(m$ even, $m \geq 2)$.

Step 3. Let $p(z, \bar{z}):=\left.|-\sqrt{2}| z\right|^{2}+z^{2}+\left.\bar{z}^{2}\right|^{2} \in \mathscr{S}^{2}$. Then there exists a (real) linear functional $\Lambda_{4}^{h}$ on $\mathbf{R}[x, y]_{4}^{h}$ such that $\Lambda_{4}^{h}(p)<$ $0,\left.\Lambda_{4}^{h}\right|_{\Gamma_{4}^{h}} \geq 0$, and $\left.\Lambda_{4}^{h}\right|_{\text {int. } \Gamma_{4}^{h}}>0$. This is done using the specific form of the polynomials in $\Gamma_{4}^{h}$ and positivity properties for $3 \times 3$ scalar matrices.

Step 4. $\mathscr{W}_{4}^{h} \subseteq \Gamma_{4}$, i.e., the $\left(1-|z|^{2}\right) \sum_{j}\left|r_{j}(z)\right|^{2}$ component of a homogeneous polynomial of total degree 4 can be eliminated.

Step 5. $\Lambda_{4}^{h}$ can be extended to a linear functional $\Lambda_{4}$ on $\mathbf{R}[x, y]_{4}$, maintaining the nonnegativity on the cone $\mathscr{W}_{4}$, and the strict positivity on the interior of $\mathscr{W}_{4}$. This is accomplished by an application of the Hahn-Banach Theorem on the finite dimensional vector space $\mathbf{R}[x, y]_{4}$, along the lines of Théorème 4 in [Cas].

Step 6. $\Lambda_{4}$ can be extended, one step at a time, to all of $\mathbf{C}[z, \bar{z}]$, maintaining the positivity on each $\mathscr{W}_{m}$, and thus giving rise to a (necessarily bounded) linear functional $\Lambda$ on $\mathbf{C}[z, \bar{z}]$, which is nonnegative on $\mathscr{W}$ and with $\Lambda(p)<0$.

It follows that $\Lambda$ separates the polynomial $p \in \mathscr{S}^{2}$ from the cone $\mathscr{W}$.

\section{CONSEQUENCES OF THE MAIN RESULT}

Corollary 1. There exists a polynomially hyponormal operator which is not subnormal.

By combining [ $\mathrm{McCP}$, Theorem 3.4] and Corollary 1, we can show that:

Corollary 2. There exists a unilateral weighted shift that is polynomially hyponormal but not subnormal.

Since the spectrum of a weighted shift is polynomially convex, it also follows that there exists an analytically hyponormal operator (i.e., with respect to the analytic functional calculus) which is not subnormal.

In the classical theory of moments, a central problem is to deduce whether a polynomial nonnegative on a certain compact set $K:=f^{-1}([0,+\infty)) \subseteq \mathbf{R}^{n}$ can be written as a combination (or 
limit of combinations) of squares of polynomials, possibly multiplied by the defining function $f$ (see [Cas, Lan]). The relevance of Theorem 1 to this problem is illustrated by the next two examples.

Corollary 3. The polynomial $\left.|-\sqrt{2}| z\right|^{2}+z^{2}+\left.\bar{z}^{2}\right|^{2}$ is not the uniform limit (on $\overline{\mathbf{D}}$ ) of polynomials of the form $\sum_{i}\left|\overline{q_{i}(z)}+\overline{p_{i}(z)} r_{i}(z)\right|^{2}+$ $\left(1-|z|^{2}\right) \cdot \sum_{j}\left|s_{j}(z)\right|^{2}, p_{i}, q_{i}, r_{i}, s_{i} \in \mathbf{C}[z]$.

Corollary 4. On $[0,1]$, not every nonnegative polynomial can be approximated in the uniform norm by polynomials of the form

$$
\begin{aligned}
& \sum_{k}\left[\sum_{i \geq 0} x^{i}\left|b_{i k}+\sum_{j} a_{i+j, k} c_{j k} x^{j}\right|^{2}\right. \\
& \left.\quad+\sum_{i \geq 1} x^{i}\left|\sum_{j} a_{j k} c_{i+j, k} x^{j}\right|^{2}+(1-x) \cdot \sum_{i} x^{i}\left|d_{i k}\right|^{2}\right],
\end{aligned}
$$

where $a_{i k}, b_{i k}, c_{i k}, d_{i k}$ are complex numbers.

Details of this work will appear elsewhere.

\section{REFERENCES}

[Ag1] J. Agler, The Arveson extension theorem and coanalytic models, Integral Equations Operator Theory 5 (1982), 608-631.

[Ag2] _ Hypercontractions and subnormality, J. Operator Theory 13 (1985), 203-217.

[Cas] G. Cassier, Problème des moments sur un compact de $\mathbf{R}^{n}$ et décomposition des polynômes a plusieurs variables, J. Funct. Anal. 58 (1984), 254-266.

[Cla] K. Clancey, Seminormal operators, Lecture Notes in Math., vol. 742, Springer-Verlag, New York, Heidelberg, Berlin, 1979.

[Con] J. Conway, Subnormal operators, Research Notes in Math., vol. 51, Pitman Books Ltd., Boston, London, Melbourne, 1981.

[Cu1] R. E. Curto, Quadratically hyponormal weighted shifts, Int. Equations Operator Theory 13 (1990), 49-66.

[Cu2] _ Joint hyponormality: A bridge between hyponormality and subnormality, Proc. Sympos. Pure Math. 51 (2) (1990), 69-91.

[CF] R. Curto and L. Fialkow, Recursively generated weighted shifts and the subnormal completion problem, preprint, 1990.

[CMX] R. Curto, P. Muhly, and J. Xia, Hyponormal pairs of commuting operators, Operator Theory Adv. Appl. 35 (1988), 1-22.

[Hal] P. R. Halmos, A Hilbert space problem book (2nd ed.), Springer-Verlag, Berlin and New York, 1982.

[Lan] H. J. Landau (ed.), Moments in mathematics, Proc. Sympos. Appl. Math., vol. 37, Amer. Math. Soc., Providence, RI, 1987. 
[MP] M. Martin and M. Putinar, Lectures on hyponormal operators, Operator Theory: Adv. Appl., vol. 39, Birkhäuser-Verlag, 1989.

[McCP] S. McCullough and V. Paulsen, A note on joint hyponormality, Proc. Amer. Math. Soc. 107 (1989), 187-195.

[Put] M. Putinar, Hyponormal operators are subscalar, J. Operator Theory 12 (1984), 385-395.

[Shi] A. Shields, Weighted shift operators and analytic function theory, Math. Surveys 13 (1974), 49-128.

[St] J. Stampfli, Which weighted shifts are subnormal, Pacific J. Math. 17 (1966), 367-379.

[Tho] J. Thomson, Approximation in the mean by polynomials, preprint, 1989.

[Xia] D. Xia, Spectral theory of hyponormal operators, Operator Theory, Adv. Appl., vol. 10, Birkhäuser, Basel, 1983.

Department of Mathematics, University of Iowa, Iowa City, Iowa 52242 E-mail address: blaaulpd@uiamvs.bitnet

Institute of Mathematics, Romanian Academy of Sciences, P. O. Box 1-764, RO 70700 BUCHAREST, ROMANIA

Current address: Department of Mathematics, University of Iowa, Iowa City, Iowa 52242

E-mail address: mputinar@ukanvax.bitnet 